\title{
Factors associated with systolic hypertension in peritoneal dialysis patients
}

\author{
Surachet Vongsanim ${ }^{1} \cdot$ Andrew Davenport ${ }^{2}$ (D)
}

Received: 14 May 2019 / Accepted: 30 July 2019 / Published online: 10 August 2019

(C) The Author(s) 2019

\begin{abstract}
Background Hypertension is common in peritoneal dialysis (PD) patients and associated with adverse outcomes. Besides solute clearance, PD convective clearance is used to control extracellular water (ECW) volume and sodium balance. Previous studies have reported on hypertension in PD patients treated with continuous ambulatory peritoneal dialysis (CAPD) using hypertonic glucose dialysates. However, increasing numbers of PD patients are now treated with automated peritoneal dialysis (APD) and icodextrin dialysates. As such, we wished to explore factors associated with systolic blood pressure (SBP) in a modern cohort to identify targets to improve blood pressure control in PD patients.

Methods We retrospectively reviewed the results from PD patients attending for peritoneal membrane assessment who had corresponding bioimpedance ECW and brain natriuretic peptide (NT-proBNP) measurements.

Results We studied 510 PD patients: 317 (72.2\%) male, 216 (42.4\%) diabetics, median age 59 (47-72) years, and 51\% treated by APD with a day-time icodextrin exchange. Mean systolic blood pressure (SBP) was $140 \pm 24.8 \mathrm{mmHg}$. SBP was independently associated with 4-hour dialysate to plasma creatinine ratio ( $\beta=29.5$ (95\% confidence limits $11.4-47.5$, $\mathrm{p}=0.001), \mathrm{N}$-terminal brain natriuretic peptide $[\beta=11.9(7.2-16.7), \mathrm{p}<0.001]$, and daily urine sodium excretion $[\beta=1.7$ $(1.0-2.3), \mathrm{p}<0.001]$.

Conclusion In the era of APD cyclers and icodextrin, SBP is associated with increased NT-proBNP, a marker of ECW expansion, and faster peritoneal transport, a risk factor for a positive sodium balance, and increased urinary sodium suggestive of higher dietary sodium intake. Patients should be encouraged to restrict sodium intake and PD prescriptions targeted to control ECW to improve SBP control.
\end{abstract}

Keywords Systolic blood pressure $\cdot$ Extracellular water $\cdot$ Sodium $\cdot$ Peritoneal transport $\cdot$ Brain natriuretic peptide

\section{Introduction}

Hypertension is one of the major determinants contributing to the increased cardiovascular morbidity and mortality in dialysis patients [1]. Moreover, hypertension is very common in patients with end-stage kidney disease treated with peritoneal dialysis (PD), affecting more than $80 \%$ of

Andrew Davenport

a.davenport@ucl.ac.uk

Surachet Vongsanim

surachet.vongsanim@nhs.net

1 Renal Division, Department of Internal Medicine, Faculty of Medicine, Chiang Mai University, 110 Intrawarorot Road, Amphoe Mueang Chiang Mai, Chiang Mai 50200, Thailand

2 UCL Department of Nephrology, Royal Free Hospital, University College London, London, UK patients and observational studies reporting an association with worse long-term outcomes [2, 3]. The prospective Netherlands Cooperative Study on the Adequacy of Dialysis observed an association between high systolic blood pressure and an increased risk of mortality [4]. Current clinical guideline from the International Society of Peritoneal Dialysis (ISPD) recommends a target blood pressure of less than 140/90 $\mathrm{mmHg}$ as the treatment goal for PD patients [5]. However, this recommendation was based mainly on studies from the general and chronic kidney disease populations, rather than specifically from studies in PD patients.

Understanding the relationship between clinical factors affecting the blood pressure is the first step to improve treatment outcomes for individual patients. Although some studies have reported an association between volume overload, with extracellular water (ECW) expansion and hypertension $[6,7]$, whereas other reports failed to demonstrate any 
association between volume overload and hypertension [8], and others observed an effect of restricting dietary sodium intake [9]. The introduction of bioimpedance devices into clinical practice to measure ECW expansion and body composition [10], has led to the realization that ECW expansion can also occur in patients with malnutrition and inflammation [11], and this may aid explaining the discordant results of previous studies investigating the association between volume status and hypertension.

Many of these previous studies investigated patients treated by continuous ambulatory peritoneal dialysis (CAPD) prescribed hypertonic glucose peritoneal dialysates. In recent years, more patients in Europe and North America are treated using automated peritoneal dialysis cyclers (APD), rather than CAPD, and APD cyclers help reducing ECW expansion in faster peritoneal transporters $[12,13]$. Similarly, the introduction of icodextrin peritoneal dialysates for the long dwell has been shown to reduce ECW expansion compared to hypertonic glucose [8].

We therefore wished to identify factors associated with systolic blood pressure in a modern day cohort of PD patients prescribed icodextrin dialysates for both CAPD and APD, that would aid understanding of pathophysiology of hypertension and generate hypotheses that could be tested and improve blood pressure control in PD patients.

\section{Study methods}

We retrospectively reviewed the results from adult peritoneal dialysis patients who had attended for routine peritoneal membrane assessment in our centre between January 2008 and October 2018. No patient had been treated for PD peritonitis or had an emergency hospital admission within the preceding 3 months. Patients with a urinary output of $\geq 200 \mathrm{~mL} /$ day were prescribed frusemide $250 \mathrm{mg}$ daily. We excluded patients who had implantable cardiac devices, amputations and those were unable to stand. Patients starting PD were provided with dietary advice to restrict dietary sodium to $100 \mathrm{mmol} /$ day.

All patients used standard low pH glucose or 7.5\% icodextrin dialysates (Baxter health Care, Deerfield, Illinois, USA). Patients were weighed and had bioimpedance measurements post voiding and with peritoneal dialysate drained out [14]. Peritoneal transport (PET) was calculated as the ratio of 4-hour peritoneal dialysate effluent creatinine to serum, using a 2 litres of $22.7 \mathrm{~g} / \mathrm{L}$ glucose exchange [13]. PD adequacy and dietary protein nitrogen appearance adjusted for body weight (nPNA) were calculated by standard methods from 24-hour urine and peritoneal dialysate effluent samples [13]. In addition to standard blood tests, we also measured $\mathrm{N}$-terminal brain natriuretic peptide (NT-proBNP). Sodium in urine and dialysates was measured using an indirect ion electrode [15]. CAPD patients were taught to allow $15 \mathrm{~s}$ for the flush before fill technique, and as such we allowed $90 \mathrm{~mL}$ to compensate for this when estimating sodium balance as the difference between the total amount of sodium instilled with PD dialysates and that measured in 24-hour PD effluent and urine collections. Multifrequency bioelectrical impedance assessments (MFBIA) were measured with an eight electrode multifrequency segmental bioimpedance device (InBody 720, Seoul, South Korea) using a standardised protocol, after the patient had drained out peritoneal dialysate. Extracellular water (ECW) and skeletal muscle mass (SMM) [16], were normalized by height and height squared, respectively, to allow comparison between patients. Patients were admitted for the peritoneal membrane assessment and blood pressure was recorded in the supine position after 4 hours when the patient had drained out their dialysate and then rested for a minimum additional $30 \mathrm{~min}$ and had abstained from any stimulants. Blood pressure measurements were repeated, and if similar the first blood pressure recorded, but if lower, then a third measurement was made, and the mean of the lower two recordings taken, following the British Society of Hypertension guidelines [17]. Blood pressure monitors (Dinamap, Critikon Corporation, Tampa, FL, USA) were regularly serviced and calibrated. Relevant medical history and echocardiography results were obtained from hospital computerised records. Left ventricular mass was calculated from 2-dimensional transthoracic echocardiograms (Philips IE33, Philips Medical Systems, Eindhoven, the Netherlands) and analysed offline by experienced observers using the equation of Devereux [18]. Left ventricular mass index (LVMI) was calculated as the left ventricular mass divided by body surface area (BSA) using the Gehan and George formula [19].

\section{Ethics}

Our retrospective audit complied with the UK National Health Service guidelines for clinical audit and service development with all patient data anonymised and complied with UK National Institute for Clinical Excellence best practices, www.nice.org.uk/media/796/23/bestpracti ceclinicalaudit.pdf.

\section{Statistical analysis}

All categorical data are presented as percentage and continuous data as mean \pm standard deviation, or median (interquartile range). Groups were compared by anova or Kruskal-wallis, for parametric and nonparametric data with appropriate post hoc testing. Univariate correlation was by Pearson or Spearman analysis for parametric and nonparametric data, respectively. Variables of interest $(p<0.1)$ were then entered into multivariable step-backward logistic regression 
model. Transformation of data was performed if required to improve variable distribution. Variables were then only retained where the $95 \%$ confidence intervals for the estimate did not include zero or there was an improvement in model fit (as demonstrated by the $-2 \log$ likelihood), models were checked for collinearity and variable inflation factor. Statistical analysis used Statistical Package for Social Science version 24.0 (IBM Corporation, Armonk, New York, USA). Statistical significance was taken as $\mathrm{p}<0.05$.

\section{Results}

Five hundred and forty-nine patients started PD, 31 patients were unable to have bioimpedance measurements and 8 patients developed PD peritonitis shortly after starting PD or had an acute illness, and so we studied five hundred and ten adult PD patients attending the clinic for their first assessment of peritoneal membrane function 2 (2-3) months after starting PD, who had MFBIA recorded on the same day; (Table 1). Antihypertensive medications were prescribed to $80.8 \%$ of patients, median number of medications 1 (1-2), and diuretics to $84.6 \%$.

Three hundred and ninety-three $(77.1 \%)$ had echocardiography reports.

We divided patients according to peritoneal transport status [13], patients who were faster transporters had higher systolic blood pressure (SBP), NTproBNP, and bothe EC and ECW adjusted for height compared to slower transporters (Table 2).

On univariate analysis SBP was positively associated with diabetes, serum creatinine, urine sodium, peritoneal membrane transporter status, NT-proBNP, ECW/height and SMM/height ${ }^{2}$ and negatively with serum albumin. We found no statistically significant association between left ventricular ejection fraction (LVEF), left ventricular mass index (LVMI) and SBP, prescription of, or the number of anti-hypertensive medications prescribed (Table 3).

A multivariable step backwards regression analysis including all factors that were significant on univariate analysis. SBP was independently associated with faster membrane transport, daily urine sodium excretion, and NTproBNP (Table 4).

\section{Discussion}

The majority of the newly established PD patients in our study were fast average or fast transporters [13], with $50.8 \%$ patients treated with APD and a day time icodextrin exchange and $71.4 \%$ prescribed icodextrin as part of their PD prescription. As such, compared to previous reports, we report on an incident cohort of PD patients with the majority
Table 1 Patient demographic characteristics

\begin{tabular}{|c|c|}
\hline Characteristics & Values \\
\hline Male (\%) & $317(72.2 \%)$ \\
\hline Age, years & $59(47-72)$ \\
\hline Diabetes mellitus (\%) & $216(42.4 \%)$ \\
\hline Icodextrin usage (\%) & $364(71.4 \%)$ \\
\hline \multicolumn{2}{|l|}{ PD mode $(\%)$} \\
\hline APD & $138(27.1 \%)$ \\
\hline CAPD & $113(22.12 \%)$ \\
\hline APD with day time exchange & $259(50.8 \%)$ \\
\hline \multicolumn{2}{|l|}{ Office blood pressure, $\mathrm{mmHg}$} \\
\hline Systolic & $140.9 \pm 24.8$ \\
\hline Diastolic & $81.5 \pm 15.3$ \\
\hline Serum creatinine, ummol/L & $566(434-742)$ \\
\hline Weekly Kt/Vurea & $2.56(2.06-3.29)$ \\
\hline Urine volume, $\mathrm{mL} / \mathrm{day}$ & $1105(573-1680)$ \\
\hline Urinary sodium loss, $\mathrm{mmol} /$ day & $59(30-156)$ \\
\hline Peritoneal sodium loss, $\mathrm{mmol} /$ day & $47.7(-195$ to 405$)$ \\
\hline PET 4-hour D/Pcreatinine & $0.73 \pm 0.13$ \\
\hline Serum albumin, $\mathrm{g} / \mathrm{L}$ & $37 \pm 4.5$ \\
\hline nPNA, g/kg/day & $0.87(0.74-1.05)$ \\
\hline NTproBNP, ng/L & $2163.0(837.2-7119.9)$ \\
\hline Serum sodium mmol/L & $139(136-141)$ \\
\hline Total body water, $\mathrm{L}$ & $37.50(31.30-43.43)$ \\
\hline ECW/height, Lkg/m & $8.98 \pm 1.71$ \\
\hline ECW/body weight $\mathrm{L} / \mathrm{kg}$ & $0.21 \pm 0.09$ \\
\hline SMM/height ${ }^{2}, \mathrm{~kg} / \mathrm{m}^{2}$ & $9.82 \pm 1.60$ \\
\hline LVEF, \% & $55.0(45.0-57.5)$ \\
\hline Left ventricular mass index, $\mathrm{g} / \mathrm{m}^{2}$ & $112.5(89.4-136.1)$ \\
\hline
\end{tabular}

Values presented as mean \pm standard deviation, median (interquartile range) or number (percentage)

Data expressed as integer, percentage, mean \pm standard deviation or median (interquartile range)

$A P D$ automated peritoneal dialysis cycler, $C A P D$ continuous ambulatory peritoneal dialysis, $P E T$ peritoneal equilibration test, $D / P 4 \mathrm{~h}$ dialysate to plasma ratio, $n P N A$ normalised protein nitrogen appearance rate, NTproBNP $\mathrm{N}$ terminal brain natriuretic peptide, $E C W$ extracellular water, $S M M$ skeletal muscle mass, $L V E F$ left ventricular ejection fraction

using icodextrin dialysates [2-4]. Icodextrin has been shown to improve volume control compared to hypertonic glucose and has been shown to have an important role in maintaining ultrafiltration, particularly for the faster transporter [20].

As expected, the majority of patients attending for their first assessment of peritoneal membrane function had residual renal function. Most of our patients had blood pressure measurements within the current ISPD guideline targets [5]. Compared to previous reports, our patients had lower LVMI and well preserved left ventricular function [21]. In view of the relatively short duration of treatment with PD, the lower levels of left ventricular hypertrophy reported in our 
Table 2 Patients divided according to peritoneal creatinine transport status

\begin{tabular}{|c|c|c|c|c|c|}
\hline Variables & Slow (15) & Slow average (119) & Fast average (235) & Fast (138) & $\mathrm{p}$ value \\
\hline SBP mmHg & $126(115-152)$ & $134(118-148)$ & $144(125-158)$ & $144(129-164)$ & 0.001 \\
\hline DBP mm Hg & $78 \pm 13$ & $81 \pm 15$ & $81 \pm 15$ & $82 \pm 15$ & 0.625 \\
\hline NTproBNP ng/L & $1750(829-3713)$ & $1412(465-4389)$ & $2140(899-6023)$ & $3691(1351-15,764)$ & $<0.001$ \\
\hline $\mathrm{Na}$ Balance mmol/day & $\begin{array}{l}-110.9(133.6 \text { to } \\
-63.8)\end{array}$ & $\begin{array}{l}-114.1(-159.3 \text { to } \\
-78.3)\end{array}$ & $\begin{array}{l}-114.3(-178.1 \text { to } \\
-70.45)\end{array}$ & $\begin{array}{l}-136.9(-175.7 \text { to } \\
-82.7)\end{array}$ & 0.302 \\
\hline ECW L & $12.1(10.6-14)$ & $13.3(11.4-16.3)$ & $15.2(13.0-17.8)$ & $15.35(13.1-17.3)$ & $<0.001$ \\
\hline $\mathrm{ECW} / \mathrm{Ht} \mathrm{L} / \mathrm{m}$ & $7.6(6.5-8.9)$ & $8.4(7.2-9.6)$ & $9.1(8.0-10.4)$ & $9.3(8.1-10.1)$ & $<0.001$ \\
\hline $\mathrm{ECW} / \mathrm{Wt} \mathrm{L/kg}$ & $0.19 \pm 0.04$ & $0.20 \pm 0.03$ & $0.21 \pm 0.04$ & $0.22 \pm 0.03$ & 0.424 \\
\hline
\end{tabular}

$S B P$ systolic blood pressure, $D B P$ diastolic blood pressure, $N T p r o B N P$ N terminal brain natriuretic peptide, $E C W$ extracellular water, $H t$ height, $W t$ weight

$\mathrm{p}$ value fast vs slow/slow average transporter

Table 3 Univariate analysis of factors associated with systolic blood pressure

\begin{tabular}{|c|c|c|}
\hline Variables & $\mathrm{R}$ & $\mathrm{p}$ value \\
\hline Diabetes & 0.105 & 0.018 \\
\hline Serum creatinine & 0.125 & 0.005 \\
\hline Urine sodium (mmol/day) & 0.171 & $<0.001$ \\
\hline 4-hour D/Pcreatinine & 0.209 & $<0.001$ \\
\hline Serum albumin $(\mathrm{g} / \mathrm{L})$ & -0.169 & $<0.001$ \\
\hline NT-proBNP (pg/mL) & 0.205 & $<0.001$ \\
\hline ECW/height $(\mathrm{kg} / \mathrm{m})$ & 0.211 & $<0.001$ \\
\hline SMM/height ${ }^{2}\left(\mathrm{~kg} / \mathrm{m}^{2}\right)$ & 0.195 & $<0.001$ \\
\hline
\end{tabular}

$\mathrm{N}$ terminal brain natriuretic peptide (NT-proBNP), extracellular water (ECW), skeletal muscle mass (SMM)

study, this most probably reflects the standard of pre-dialysis care provided by a specialist clinical service, designed to prepare patients for dialysis. Although LVMI is associated with hypertension, we could not find any significant association with systolic blood pressure, diastolic blood pressure, pulse pressure, or mean arterial pressure. Other studies in PD patients have similarly found no association [22]. We used clinic blood pressures in our analysis, differing from some of the previous studies, but even those using ambulatory blood pressure recordings have reported no association between LVM and blood pressure [22]. Whereas studies in haemodialysis patients have reported on blood pressure variability, similar studies using ambulatory blood pressure monitoring have not shown such day to day variability [23].

On univariate analysis, we observed a negative association between SBP and serum albumin, whereas SBP was positively correlated with diabetes, serum creatinine, 24-hour urine sodium excretion, peritoneal membrane transport status, NT-proBNP, ECW/height and SMM/ height ${ }^{2}$. After adjustment using a multivariable model, SBP was found to be independently associated with daily urine sodium excretion, 4-hour peritoneal membrane creatinine transport, and NT-proBNP.

Overhydration and hypertension are commonly reported in PD patients, and cardiovascular death is the commonest cause of mortality for PD patients [6, 24]. Volume overload has been shown to be an important factor associated with hypertension in PD patients, as better volume control has been observed to improve blood pressure control and reduce left ventricular hypertrophy $[25,26]$. NT-proBNP is a cardiac biomarker secreted by cardiomyocytes in the ventricles in response to plasma volume expansion, and increased
Table 4 Multivariable model for systolic blood pressure

\begin{tabular}{|c|c|c|c|c|c|c|}
\hline Variables & $\beta$ & $\mathrm{StE} \beta$ & St $\beta$ & $\mathrm{t}$ & $95 \% \mathrm{CI}$ & $\mathrm{p}$ value \\
\hline Diabetes mellitus & 4.15 & 2.34 & 0.09 & 1.77 & -0.46 to 8.75 & 0.08 \\
\hline Urine sodium & 1.65 & 0.34 & 0.246 & 4.86 & $0.98-2.32$ & $<0.001$ \\
\hline 4-hr D/Pcreatinine & 29.46 & 9.17 & 0.159 & 3.21 & $11.44-47.48$ & 0.001 \\
\hline NT-proBNP pmol/L & 11.90 & 2.42 & 0.243 & 4.93 & $7.15-16.65$ & $<0.001$ \\
\hline $\mathrm{SMM} / \mathrm{height}^{2} \mathrm{~kg} / \mathrm{m}^{2}$ & 1.35 & 0.77 & 0.089 & 1.758 & -0.16 to 2.87 & 0.08 \\
\hline
\end{tabular}

Nonparametric data was transformed to obtain normal distribution by log transformation (NT-proBNP) or square root (Urinary sodium). Standard error $\beta$ (StE $\beta)$, Standardized $\beta$ (St $\beta$ ), 95\% confidence intervals (95\% CI). Model fit $\mathrm{r}^{2}=0.173$, adjusted $\mathrm{r}^{2} 0.162$

$N T$-proBNP N terminal brain natriuretic peptide, SMM skeletal muscle mass 
ECW $[27,28]$. Studies in PD patients have reported an association between increasing NT-proBNP and ECW volume expansion and mortality $[27,28]$. The association with SBP would support ECW being a contributor to an increased SBP.

Diabetes is a common comorbidity in patients with chronic kidney disease. Diabetic patients may have faster transport status [29], as hyperglycaemia can increase local blood flow by protein kinase $\mathrm{C}$ mediated vasodilatation. Faster peritoneal transporters are at risk of lower ultrafiltration volumes when longer dwell cycles are prescribed due to loss of the osmotic glucose gradient. Other studies have shown that diabetic patients have ECW expansion compared to non-diabetics, even after adjusting for transporter status [30]. Although the hyperglycaemic state may reduce the osmotic effects of the dialysate glucose and so reduce convective peritoneal sodium removal, diabetic patients have also been reported to have higher tissue sodium levels [31], and this may additionally contribute to an increased risk for hypertension.

Faster peritoneal membrane permeability has been reported associated with increased mortality, possibly due to ECW expansion [20]. Although faster transport status was associated with PD technique failure and increased mortality, this was in the era before APD cyclers and icodextrin [32]. Our study had a high proportion of patients prescribed icodextrin and APD cyclers, and yet we found that faster transport was still associated with increased SBP, ECW expansion and raised NTproBNP. Although APD cyclers allow faster transporters to achieve greater ultrafiltration, the shorter cycle dwell times result in a relative greater water transport through aquaporin channels compared to sodium removal by active $\mathrm{Na} / \mathrm{K}$ ATPase transporters and $\mathrm{Na}$ cotransporters, so increasing the risk of sodium retention and systolic hypertension [33].

24-hour urine sodium has been used to estimate daily sodium intake particularly in patients with chronic kidney disease, on the basis that if patients are in neutral balance, then urinary sodium excretion should mirror dietary intake. However, this assumes that patients are in neutral balance and that 24-hour collections are both accurate and reliable. Dietary recall may also be inaccurate due to the increasing consumption of ready meals, and additions from preprepared sauces $[34,35]$. As such we were unable to reliably estimate dietary sodium intake, and sodium balance in terms of dietary intake and urinary and peritoneal sodium removal. Previous studies have demonstrated that reduced sodium intake led to a significantly lower blood pressure in PD patients [9]. On the other hand one study reported that patients with faster transport had lower peritoneal sodium losses and higher SBP [32]. This is most likely due to our incident patients having greater dietary sodium intake, whereas in the study from Turkey of prevalent patients, using only glucose dialysates, failure to achieve adequate peritoneal sodium removal led to increased SBP and volume overload. As our patients with greater sodium losses had greater muscle mass, suggesting greater sodium intake in keeping with previous studies reporting greater survival for those patients with the highest sodium removal [36], and greater mortality for those with lowest dietary sodium intake [37].

Our study was a cross sectional observational study in an incident cohortand as such we cannot attribute causality as to which factors increase SBP, but provide hypotheses which require testing. However the association with increased NTproBNP and ECW is suggestive that increased blood pressure was volume related, particularly when considering that our faster peritoneal transporters have higher SBP, NT-proBNP and ECW.

Our study of PD patients in the modern era of APD cyclers and icodextrin supports earlier reports that SBP in $\mathrm{PD}$ patients is associated with faster peritoneal transport, which is a risk factor for increased ECW and sodium retention. In our incident cohort this increased blood pressure has a volume component most likely due to increased dietary sodium intake as suggested by greater total peritoneal and urinary sodium losses, whereas in prevalent cohorts volume dependent hypertension may follow failure to achieve adequate peritoneal ultrafiltration and sodium removal [32]. Our study supports and association between ECW expansion and SBP.

Acknowledgements Surachet Vongsanim was in receipt of an International Society of Nephrology/Kidney Research UK training scholarship.

\section{Compliance with ethical standards}

Conflict of interest The authors declare that they have no conflict of interest.

Research incloving human and animal participants Our retrospective audit complied with the United Kingdom National Research Ethics guidelines for clinical audit and service development with all patient data anonymised and complied with UK National Institute for Clinical Excellence best practices, https://researchsupport.admin.ox.ac.uk/sites /default/files/researchsupport/documents/media/defining-research.pdf.

Informed consent For this type of study individual informed consent is not required.

Open Access This article is distributed under the terms of the Creative Commons Attribution 4.0 International License (http://creativeco mmons.org/licenses/by/4.0/), which permits unrestricted use, distribution, and reproduction in any medium, provided you give appropriate credit to the original author(s) and the source, provide a link to the Creative Commons license, and indicate if changes were made. 


\section{References}

1. Locatelli F, Covic A, Chazot C et al (2004) Hypertension and cardiovascular risk assessment in dialysis patients. Nephrol Dial Transplant 19(5):1058-1068

2. Cocchi R, Degli Esposti E, Fabbri A et al (1999) Prevalence of hypertension in patients on peritoneal dialysis: results of an Italian multicentre study. Nephrol Dial Transpl 14(6):1536-1540

3. Udayaraj UP, Steenkamp R, Caskey FJ et al (2009) Blood pressure and mortality risk on peritoneal dialysis. Am J Kidney Dis 53(1):70-78

4. Jager KJ, Merkus MP, Boeschoten EW et al (1999) Dialysis in The Netherlands: the clinical condition of new patients put into a European perspective. NECOSAD study group. Netherlands cooperative study on the adequacy of dialysis phase 1 . Nephrol Dial Transpl 14(10):2438-2444

5. Wang AY, Brimble KS, Brunier G et al (2015) ISPD cardiovascular and metabolic guidelines in adult peritoneal dialysis patients part I-assessment and management of various cardiovascular risk factors. Perit Dial Int 35(4):379-387

6. Koc M, Toprak A, Tezcan H et al (2002) Uncontrolled hypertension due to volume overload contributes to higher left ventricular mass index in CAPD patients. Nephrol Dial Transplant 17(9):1661-1666

7. Konings CJ, Kooman JP, Schonck M et al (2003) Effect of icodextrin on volume status, blood pressure and echocardiographic parameters: a randomized study. Kidney Int 63(4):1556-1563

8. Van Biesen W, Williams JD, Covic AC et al (2011) Fluid status in peritoneal dialysis patients: the European body composition monitoring (EuroBCM) study cohort. PLoS One 6(2):e17148

9. Inal S, Erten Y, Tek N et al (2014) The effect of dietary salt restriction on hypertension in peritoneal dialysis patients. Turk $\mathrm{J}$ Med Sci 44(5):814-819

10. Davies SJ, Davenport A (2014) The role of bioimpedance and biomarkers in helping to aid clinical decision-making of volume assessments in dialysis patients. Kidney Int 86(3):489-496

11. Demirci MS, Demirci C, Ozdogan O et al (2011) Relations between malnutrition-inflammation-atherosclerosis and volume status. The usefulness of bioimpedance analysis in peritoneal dialysis patients. Nephrol Dial Transpl 26(5):1708-1716

12. Brown EA, Davies SJ, Rutherford P et al (2003) Survival of functionally anuric patients on automated peritoneal dialysis: the European APD outcome study. J Am Soc Nephrol 14(11):2948-2957

13. van Biesen W, Heimburger O, Krediet R et al (2010) Evaluation of peritoneal membrane characteristics: clinical advice for prescription management by the ERBP working group. Nephrol Dial Transpl 25(7):2052-2062

14. Davenport A (2013) Effect of intra-abdominal dialysate on bioimpedance-derived fluid volume status and body composition measurements in peritoneal dialysis patients. Perit Dial Int 33(5):578-579

15. Persaud J, Thomas M, Davenport A (2014) Indirect ion selective electrode methods potentially overestimate peritoneal dialysate sodium losses. Ther Apher Dial 18(4):321-325

16. Furstenberg A, Davenport A (2011) Assessment of body composition in peritoneal dialysis patients using bioelectrical impedance and dual-energy x-ray absorptiometry. Am J Nephrol 33(2):150-156

17. Williams B, Poulter NR, Brown MJ et al (2004) BHS guidelines working party, for the british hypertension society. British hypertension society guidelines for hypertension management 2004 (BHS-IV): summary. Brit Med J 328(7440):634-640

18. Devereux RB, Alonso DR, Lutas EM et al (1986) Echocardiographic assessment of left ventricular hypertrophy: comparison to necropsy findings. Am J Cardiol 57(6):450-458
19. Gehan EA, George SL (1970) Estimation of human body surface area from height and weight. Cancer Chemother Rep 54(4):225-235

20. Davies SJ, Woodrow G, Donovan K et al (2003) Icodextrin improves the fluid status of peritoneal dialysis patients: results of a double-blind randomized controlled trial. J Am Soc Nephrol 14(9):2338-2344

21. Foley RN, Parfrey PS, Harnett JD et al (1995) Clinical and echocardiographic disease in patients starting end-stage renal disease therapy. Kidney Int 47(1):186-192

22. Konings CJ, Kooman JP, Schonck M et al (2002) Fluid status, blood pressure, and cardiovascular abnormalities in patients on peritoneal dialysis. Perit Dial Int 22(4):477-487

23. Tonbul Z, Altintepe L, Sözlü C et al (2002) Ambulatory blood pressure monitoring in haemodialysis and continuous ambulatory peritoneal dialysis (CAPD) patients. J Hum Hypertens 16(8):585-589

24. Chen YC, Lin CJ, Wu CJ et al (2009) Comparison of extracellular volume and blood pressure in hemodialysis and peritoneal dialysis patients. Nephron Clin Pract 113(2):c112-c116

25. Sav T, Oymak O, Inanc MT et al (2009) Effects of twice-daily icodextrin administration on blood pressure and left ventricular mass in patients on continuous ambulatory peritoneal dialysis. Perit Dial Int 29(4):443-449

26. Luo YJ, Lu XH, Woods F et al (2011) Volume control in peritoneal dialysis patients guided by bioimpedance spectroscopy assessment. Blood Purif 31(4):296-302

27. Davenport A (2012) Changes in N-terminal pro-brain natriuretic peptide correlate with fluid volume changes assessed by bioimpedance in peritoneal dialysis patients. Am J Nephrol 36(4):371-376

28. Paniagua R, Ventura MD, Avila-Diaz M et al (2010) NT-proBNP, fluid volume overload and dialysis modality are independent predictors of mortality in ESRD patients. Nephrol Dial Transpl 25(2):551-557

29. Lin JJ, Wadhwa NK, Suh H et al (1995) Increased peritoneal solute transport in diabetic peritoneal dialysis patients. Adv Perit Dial 11:63-66

30. Udo A, Goodlad C, Davenport A (2017) Impact of diabetes on extracellular volume status in patients initiating peritoneal dialysis. Am J Nephrol 46(1):18-25

31. Kopp C, Linz P, Maier C et al (2018) Elevated tissue sodium deposition in patients with type 2 diabetes on hemodialysis detected by (23) Na magnetic resonance imaging. Kidney Int 93(5):1191-1197

32. Ates K, Nergizoglu G, Keven K et al (2001) Effect of fluid and sodium removal on mortality in peritoneal dialysis patients. Kidney Int 60(2):767-776

33. Struijk DG, Krediet RT (2000) Sodium balance in automated peritoneal dialysis. Perit Dial Int 20(Suppl 2):S101-S105

34. Amalia RI, Davenport A (2019) Estimated dietary sodium intake in peritoneal dialysis patients using food frequency questionnaires and total urinary and peritoneal sodium losses and assessment of extracellular volumes. Eur J Clin Nutr 73(1):105-111

35. Gkza A, Davenport A (2017) Estimated dietary sodium intake in haemodialysis patients using food frequency questionnaires. Clin Kidney J 10(5):715-772

36. Wang X, Axelsson J, Lindholm B et al (2005) Volume status and blood pressure in continuous ambulatory peritoneal dialysis patients. Blood Purif 23(5):373-378

37. Dong J, Li Y, Yang Z, Luo J (2010) Low dietary sodium intake increases the death risk in peritoneal dialysis. Clin J Am Soc Nephrol 5(2):240-247

Publisher's Note Springer Nature remains neutral with regard to jurisdictional claims in published maps and institutional affiliations. 